\title{
Prediction of Lymphohematopoietic Diseases by Incidentally- Detected Diffuse Signal Alterations of Bone Marrow on MRI
}

Rafiye Ciftciler', [MD]

ORCID: 0000-0001-5687-8531

Kader Karli Oguz ${ }^{2}$ [MD]

ORCID: 0000-0002-3385-4665

Yahya Buyukasik', [MD]

ORCID: 0000-0002-4764-2348

\section{n ABSTRACT Con}

Objective: Advanced imaging techniques are increasingly used in the diagnostic workup of patients. The clinical significance of incidentallydetected signal alterations of the bone marrow on magnetic resonance imaging has not been widely studied. The purpose of this study was to determine whether bone marrow signal changing on magnetic resonance imaging could predict a hematologic disease.

Materials and Methods: Thirty patients who were referred to Hematology department due to bone marrow signal alteration on magnetic resonance imaging between the years of 2011 and 2018 were evaluated.

Results: There were 8 males and 22 females with a median age of 53 (range, 31-86) years at the time of presentation to the Hematology clinic. The magnetic resonance imaging studies had been taken because the patients had complaints of pain in extremities or lumbago (80\%). The patients had been followed for median 4.5 months (0-71.7). Six (20\%) cases had a bone marrow biopsy at presentation and a diagnosis was established in $5(16.6 \%)$ of them ( 1 patient was diagnosed acute myeloid leukemia, 4 patients were diagnosed multiple myeloma). Marrow biopsy was done in 11 of the cases during follow-up median 3.8 months (1.311.5) after presentation. A diagnosis was made in 9 cases $(5$ patients were diagnosed multiple myeloma, 2 patients were diagnosed follicular lymphoma, 1 patient was diagnosed waldenstrom macroglobulinemia). Thirteen cases never had a biopsy. These cases had been followed for 1.3 months (0-71.7). None of them showed clinical abnormalities related to a lymphohematopoietic disorder and/or diagnosed with such a disorder. Only 1 patient was diagnosed with osteomalacia at follow-up.

Conclusion: Incidentally-detected signal alterations of the bone marrow on magnetic resonance imaging should trigger investigations for a marrow problem. Most of the diagnosis patients were multiple myeloma. Extremity pain and findings like anemia may be associated with lymphohematological malignancies.

Keywords: Bone marrow, hematologic disease, magnetic resonance imaging

\section{INRODUCTION}

Advanced imaging techniques, such as magnetic resonance imaging (MRI), are increasingly used in the diagnostic workup of patients. As a result, physicians are also able to visualize the patients' bone marrow on MRI [1]. The bone marrow is to be composed of fat, water and cellular elements supported by reticulum cells, nerves, and vessels. Red marrow is involved in the production of red blood cells, white blood cells and platelets. Yellow marrow is hematopoietically inactive and composed of fat cells [2]. The difference in composition of yellow and red marrow, the latter 
containing more water and less fat, explains the appearance of the bone marrow on MRI. Changes in the composition of bone marrow can be recognized and might be the first sign of disease [3, 4]. Normal marrow should be isointense to hyperintense relative to muscle, whereas pathologic marrow is hypo-intense on T1weighted images [2]. Incidental abnormalities in signal intensity of the bone marrow may be observed in routine imaging $[5,6]$. Incidentally detected signal alterations of the bone marrow on MRI are radiologically significant, for this reason they are reported by radiologists. The clinical significance of incidentally detected signal alterations of the bone marrow on MRI is unknown. It is not known if patients with signal alteration on MRI may have hematologic disease. The purpose of this study was to determine whether bone marrow signal changing on MRI could predict a hematologic disease.

\section{MATERIAL and METHODS}

\section{Study Design and Data Collection}

Adult ( $\geq 18$ years) patients who were referred to Hacettepe University Faculty of Medicine Department of Hematology for incidentally found bone marrow signal alteration between 2011 and 2018 were searched in our department electronically database. This database is used for routine patient records and it is maintained prospectively. Details of MRI were obtained from the hospital database. Patients with obvious clinical abnormalities related to a lymphohematopoietic disorder were excluded. MRI performed at 1.5 Tesla scanner. All patients had T1-weighted spinecho (TR/TE; 550-600/15-20 msec) at least at two orthogonal planes and sagittal T2- weighted turbo spin echo (TR/TE; 3000/90 msec) imaging. All of the ethical considerations had been strictly followed in accordance with the 1964 Helsinki declaration. As a standard care/action of the hospitals of the Hacettepe Medical School, it has been recognized from the patient records that all of the studied patients had given informed consents at the time of hospitalization and before the administration of diagnostic/therapeutic standard of care.

\section{Statistical Analysis}

Demographic characteristics were presented using proportions and medians (range) for categorical and continuous variables, respectively. The statistical analyses were performed using SPSS v25.

\section{RESULTS}

\section{Characteristics of the Study Population}

A total of 30 patients were found. Patient characteristics are summarized in Table 1. There were 8 males and 22 females with a median age of 53 (range, 31-86) years at the time of presentation to the Hematology Clinic. The MRI studies had been taken because the patients had complaints of pain in extremities or lumbago (80\%). The MRIs performed were of the shoulder, knee, cranium, pelvis and mostly spine. Most of patients had no known comorbidities at the time of presentation (40\%). In $4(13.3 \%)$ of the patients with multiple myeloma diagnosis, focal lytic bone lesions at different locations were detected at the later MRI. No plasmacytoma was detected patients with multiple myeloma diagnosis. The general characteristics of the patients are shown in Table 1.

\section{Prevalence of Hematological Disorders}

The patients had been followed for median 4.5 months (0-71.7). We performed a biopsy immediately or in the follow up according to the clinical presentation and symptoms of the patients. Six $(20 \%)$ cases had a bone marrow biopsy at presentation and a diagnosis was established in 5 (16.6\%) of them (1 patient was diagnosed acute myeloid leukemia, 4 patients were diagnosed multiple myeloma). Marrow biopsy was done in 11 of the cases during follow-up median 3.8 months (1.3-11.5) after presentation. A diagnosis was made in 9 cases (multiple myeloma: 5 patients, follicular lymphoma: 2 patients, waldenstrom macroglobulinemia: 1 patient). Most of the diagnosis patients were multiple myeloma. Thirteen cases never had a biopsy. These cases had been followed for 1.3 months (0-71.7). None of them showed clinical abnormalities related to a lymphohematopoietic disorder and/or diagnosed with such a disorder. Only 1 patient was diagnosed with osteomalacia at follow-up. In total 14 (46.7\%) had or developed a disease which could explain the MRI findings. Median time from MRI and the diagnosis was 2 months (0.3-11.5). Four of the cases are shown in Figures 1 and 2. When we compared the patients with and without hematological 
Table 1. General characteristics of the patients

\begin{tabular}{|c|c|}
\hline Variable & N (\%) \\
\hline Patients & 30 \\
\hline Age (range) years & 53 (range, 31-86) \\
\hline Male/female & $8 / 22(26.7 \% / 73.3 \%)$ \\
\hline \multicolumn{2}{|l|}{ Personal History of Disease } \\
\hline Thyroid Disease & $2(6.7 \%)$ \\
\hline Pancreas Cancer & $1(3.3 \%)$ \\
\hline Anemia & $2(6.7 \%)$ \\
\hline Ankylosing Spondylitis & $1(3.3 \%)$ \\
\hline Epilepsy & $2(6.7 \%)$ \\
\hline Dementia & $1(3.3 \%)$ \\
\hline Coronary Artery Disease & $1(3.3 \%)$ \\
\hline Hypertension & $3(10 \%)$ \\
\hline Asthma & $1(3.3 \%)$ \\
\hline Diabetes Mellitus & $2(6.7 \%)$ \\
\hline Anorexia Nervosa & $1(3.3 \%)$ \\
\hline Scleroderma & $1(3.3 \%)$ \\
\hline No known diseases & $12(40 \%)$ \\
\hline \multicolumn{2}{|l|}{ MRI (n, \%) } \\
\hline Spine & $21(70 \%)$ \\
\hline Shoulder & $2(6.7 \%)$ \\
\hline Pelvis & $1(3.3 \%)$ \\
\hline Knee & $1(3.3 \%)$ \\
\hline Cranium & $4(13.3 \%)$ \\
\hline Nasopharynx & $1(3.3 \%)$ \\
\hline \multicolumn{2}{|l|}{ Complaints } \\
\hline Pain of extremities or lumbago & $24(80 \%)$ \\
\hline Syncope & $3(10 \%)$ \\
\hline Signs of infection & $3(10 \%)$ \\
\hline \multicolumn{2}{|l|}{ Diagnoses (n, \%) } \\
\hline Osteomalacia & $1(3.3 \%)$ \\
\hline Multiple Myeloma & $9(30 \%)$ \\
\hline Leukemia (Acute myeloid leukemia) & $1(3.3 \%)$ \\
\hline Lymphoma (Folliculer lymphoma) & $2(6.7 \%)$ \\
\hline Waldenstrom macroglobulinemia & $1(3.3 \%)$ \\
\hline No disease detected & $16(53.3 \%)$ \\
\hline
\end{tabular}

disease at follow-up, a significant difference was observed in terms of age $(p=0.02)$ and gender $(p=0.007)$ of the patients. There was no significant difference between the two groups in terms of complaints $(p=0.54)$. Among the laboratory values measured, a statistically significant low hemoglobin value was observed in the group with disease during follow-up ( $p=0.07)$. The clinical or laboratory characteristics of the patients who were diagnosed with a hematological disease and were not diagnosed any disease is depicted in Table 2.

\section{DISCUSSION}

Our results showed that, pain in different extremities or lumbago are the most common cause of MRI in our patient cohort. The MRIs performed were of the bony structure itself such as shoulder, knee, pelvis, or region including the bony structures in the field of view such as cranium and spine. The patients in this study had no significant clinical abnormalities associated with lymphohematopoietic disorder. Sixteen patients did not have or develop any marrow problem over time. But 13 patients were diagnosed with hematopoietic diseases and 1 patient was diagnosed with osteomalacia. In total $14(46.7 \%)$ had or developed a disease which could explain the MRI findings. The patients had been followed for median 4.5 months (0-71.7). Median time from MRI imaging and the diagnosis was 2 months (0.3-11.5).

Hematopoietic requirements in the adult normally are met by the red marrow existing in the axial skeleton, proximal femur and humerus [7]. The ratio of fat within the red marrow progressively increases with age. The mixture of fat and water component results in each contributing significantly to signal intensity. The purpose of this study was to determine whether bone marrow signal changing on magnetic resonance imaging could predict a hematologic disease. Disler et al showed that inphase and out of-phase gradient-echo MR imaging was helpful in predicting the likelihood of neoplastic or non-neoplastic signal abnormality in bone marrow [8]. Deutsch et al. followed patients for 4-15 months who received a routine MRI of the knee that showed diffuse bone marrow abnormalities. They concluded that the abnormalities seen on MRI would not lead to a hematologic disease in time [9]. Swartz et al. presented a case which was a postinflammatory cause resulting in marrow fibrosis that mimicked neoplastic marrow infiltration and necessitated biopsy for definitive diagnosis but came up as a false-positive on MRI scans [10]. In order to distinguish between benign and malignant infiltration, several contrast agents were studied using dynamic sequences as well as normal postcontrast T1-weighted sequences. A study done by Rahmouni et al, demonstrated that dynamic contrastimaging, maximal enhancement, slope, and washout were significantly different in 42 patients between low and high-grade disease found on histology. The study also show that these findings were significantly different in these patients with 
Table 2. The clinical or laboratory characteristics of the patients who were diagnosed with a hematological disease and were not diagnosed any disease

\begin{tabular}{|l|c|c|c|}
\hline & $\begin{array}{c}\text { Patients who were diagnosed } \\
\text { with a hematological disease }\end{array}$ & $\begin{array}{c}\text { Patients who were not } \\
\text { diagnosed any disease }\end{array}$ & p \\
\hline Number $(\mathrm{n})$ & $14(46.7 \%)$ & $16(53.3 \%)$ & 0.02 \\
\hline Age (range) years & 59 (range, 36-86) & $43($ range, 31-77) & 0.007 \\
Male/female & $7 / 7(50 \% / 50 \%)$ & $1 / 15(6.3 \% / 93.8 \%)$ & 0.54 \\
Complaints & $13(92.9 \%)$ & $11(68.8 \%)$ & \\
Pain of extremities or lumbago & 0 & $3(18.6 \%)$ & \\
Syncope & $1(7.1 \%)$ & $2(12.6 \%)$ & 0.07 \\
Signs of infection & $9.2(8-13,4)$ & $13(12-15.6)$ & 0.23 \\
Hemoglobin (gr/dl) (range) & $3.5 \times 10^{3} / \mu \mathrm{l}\left(0.7-20 \times 10^{3} / \mu \mathrm{l}\right)$ & $4.5 \times 10^{3} / \mu \mathrm{l}\left(3.5-12.5 \times 10^{3} / \mu \mathrm{l}\right)$ & 0.40 \\
\hline Leukocyte (range) & $120 \times 10^{3} / \mu \mathrm{l}\left(40-250 \times 10^{3} / \mu \mathrm{l}\right)$ & $150 \times 10^{3} / \mu \mathrm{l}\left(120-450 \times 10^{3} / \mu \mathrm{l}\right)$ & 0.32 \\
\hline Thrombocyte (range) & $180(65-550)$ & $150(40-250)$ & \\
LDH (u/lt) (range) & & & \\
\hline
\end{tabular}

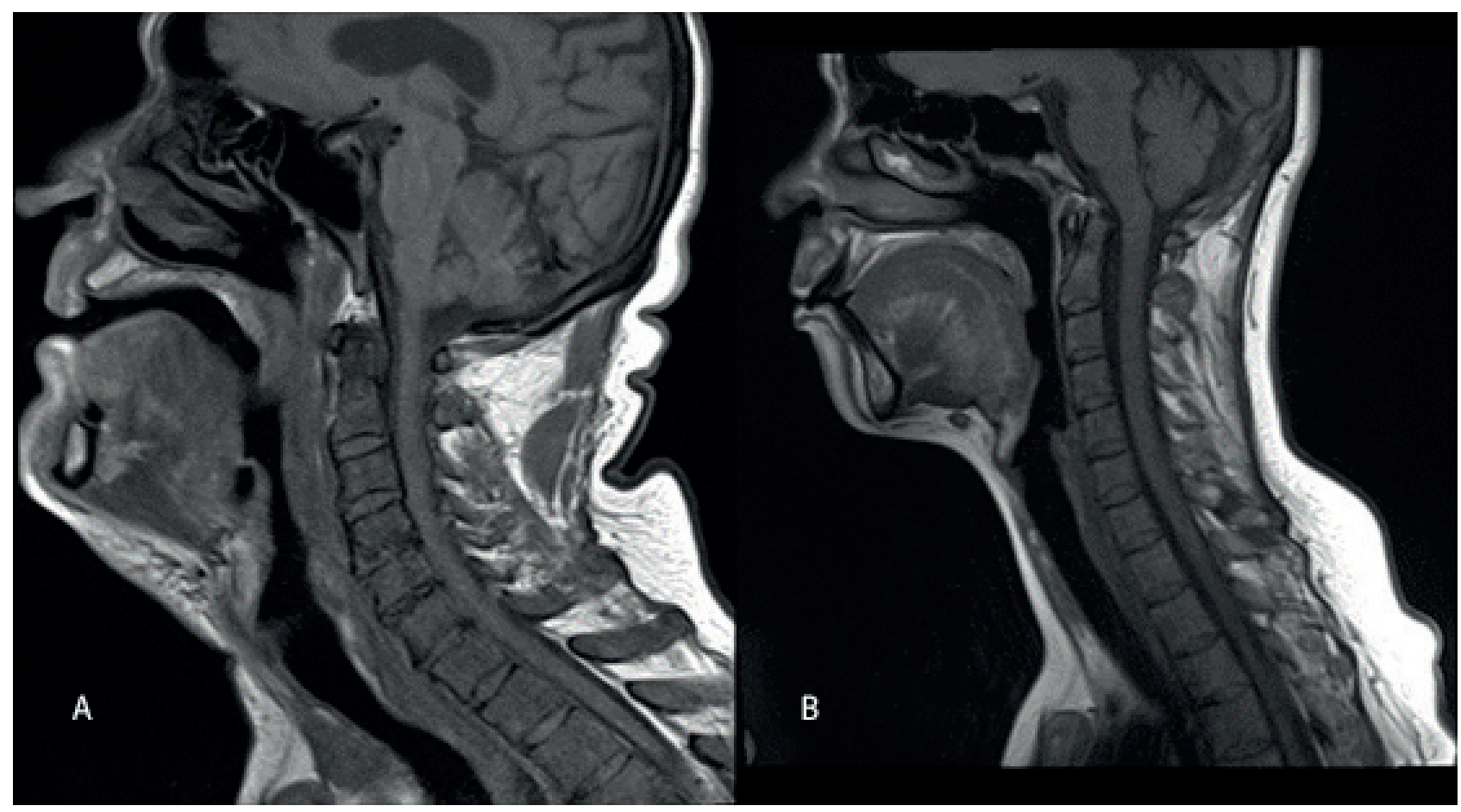

Figure 1. A, 86 years old man with deep neck infection, Sagittal T1weighted image of cervical spine shows bone marrow signal alterations, he was diagnosed acute myeloid leukemia with bone marrow biopsy (the bone marrow is diffusely abnormal). B, 36 years old woman with neck pain and lumbago, signal alterations in the bone marrow that are suspicious in terms of infiltrative diseases, she was diagnosed osteomalacia at follow up (the bone marrow is diffusely abnormal).

proven marrow infiltration compared with normal subjects [11]. In early stage multiple myeloma and monoclonal gammopathy of unknown significance, findings on MRI correlate with earlier onset of more aggressive disease, especially using dynamic MRI techniques [12-15]. Most of the diagnosis patients were multiple myeloma. Patients with diffuse signal alterations detected as incidental of bone marrow on MRI should be examined especially for multiple myeloma.
No specific advanced imaging techniques were used for bone marrow in this study. The reason for this is the use of routine imaging modalities such as nonspecific pain. However, T1-weighted and STIR, which are most sensitive to the imaging of bone marrow between routine imaging sequences, were taken in different planes [16]. In this study, 9 patients who are previously detected diffuse signal alterations in the bone marrow received multiple myeloma diagnosis. With the increased use of 


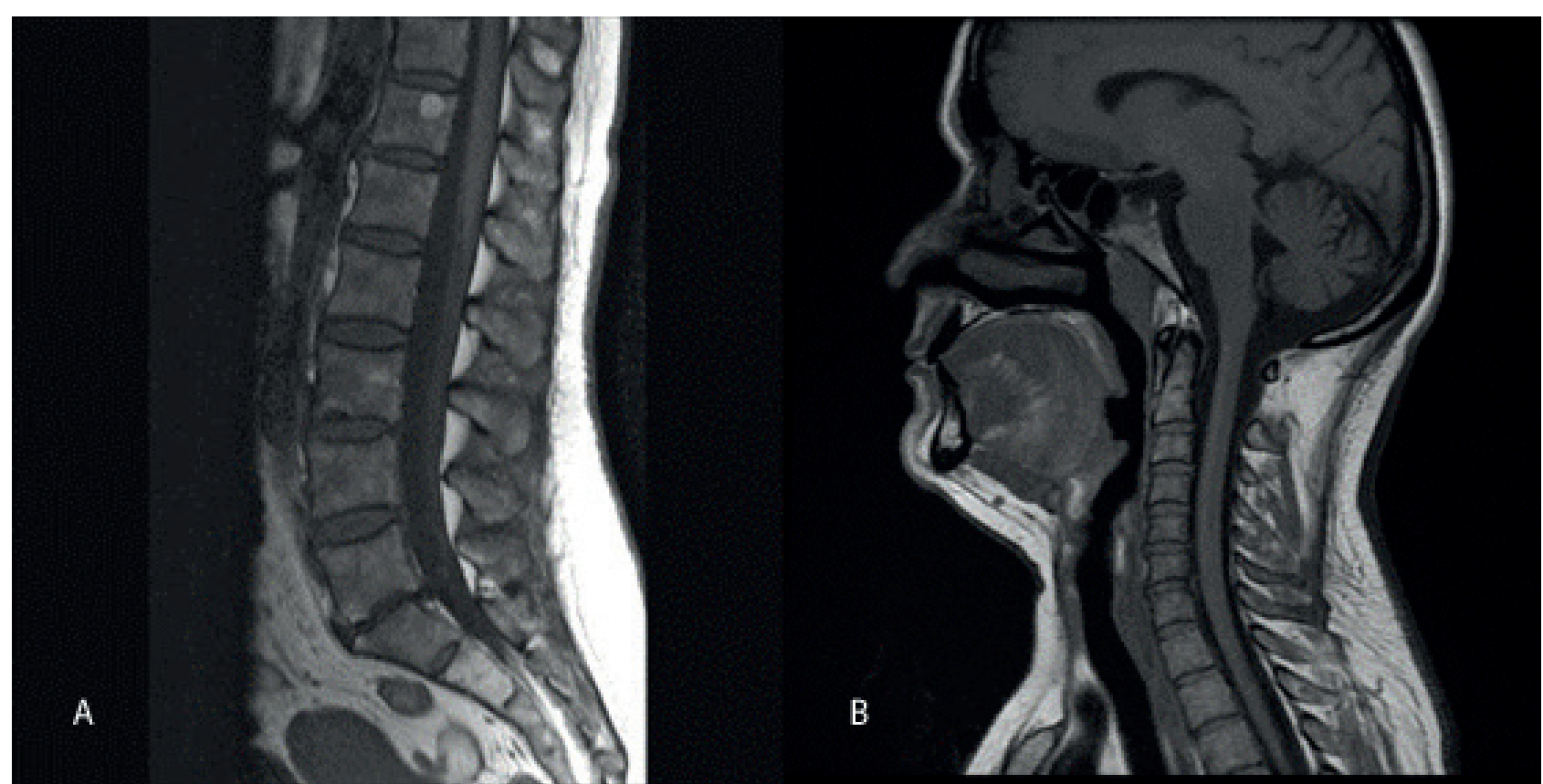

Figure 2. A, 42 years old woman with lumbago, Sagittal T1weighted image of lumbar spine shows bone marrow signal alterations, she was not diagnosed any a lymphohematopoietic disorder at follow up (the bone marrow is diffusely abnormal). B, 53 years old woman with nasopharynx infection, Sagittal T1 weighted image of cervical spine shows bone marrow signal alterations, she was not diagnosed any a lymphohematopoietic disorder at follow up (the bone marrow is diffusely abnormal).

MR for evaluation of pain complaints of patients, incidental detection of bone marrow abnormalities probably will be experienced increasingly. Many disorders can cause bone marrow signal alterations in the extremities and axial skeleton. MRI findings alone cannot usually determine the underlying condition. As a result, association with clinical history, biochemical markers, and other imaging modalities are required. In many cases, biopsy is inevitable for diagnosis. This study had some limitations. First of all the study was retrospective. There were few patients in this study. Further studies are necessary in order to conclude bone marrow signal changing in MRI could predict a hematologic disease. Until data of prospective studies are available, a bone marrow biopsy and aspiration in patients with incidentally-detected diffuse signal alterations should be considered to exclude hematologic and other diseases.
In conclusion early detection of bone marrow abnormalities may be important in the future to determine the early diagnosis of disease, treatment strategies and enhancement of outcome. Incidentally-detected signal alterations of the bone marrow on MRI should trigger investigations for a marrow problem. Extremity pain and findings like anemia may be associated with lymphohematological malignancies.

\section{CONFLICT Of INTERESTS}

The authors of this paper have no conflict of interests, including specific financial interests, relationships, and/or affiliations relevant to the subject matter or materials included. 
[1] Spierings, J, Linden AN, Kuijper PHM, et al. Incidentally detected diffuse signal alterations of bone marrow on MRI: is bone marrow biopsy indicated? Knee. 2014; 10: 67.

[2] Kung, JW, Yablon CM, Eisenberg RL. Bone marrow signal alteration in the extremities. American Journal of Roentgenology, 2011; 196(5): 492-510.

[3] Berg BCV, Malghem J, Lecouvet EF, et al. Magnetic resonance imaging of the normal bone marrow. Skeletal radiology. 1998; 27(9): 471-483.

[4] Berg, BVC, Lecouvet EF, Michaux L, et al. Magnetic resonance imaging of the bone marrow in hematological malignancies. European radiology. 1998; 8(8): 1335-1344.

[5] Ollivier L, Gerber S, Vanel D, et al. Improving the interpretation of bone marrow imaging in cancer patients. Cancer Imaging. 2006; 6(1): 194.

[6] Berg BCV, Malghem J, Lecouvet EF, et al. Classification and detection of bone marrow lesions with magnetic resonance imaging. Skeletal Radiology. 1998; 27(10): 529545.

[7] Dooms G, Fisher MR, Hricak H, et al. Bone marrow imaging: magnetic resonance studies related to age and sex. Radiology. 1985; 155(2): 429-432.

[8] Disler D, McCauley TR, Ratner LM, et al. In-phase and out-of-phase MR imaging of bone marrow: prediction of neoplasia based on the detection of coexistent fat and water. American Journal of Roentgenology. 1997; 169(5): 1439-1447.

[9] Deutsch A, Mink JH, Rosenfelt FP, et al. Incidental detection of hematopoietic hyperplasia on routine knee MR imaging. American Journal of Roentgenology. 1989; 152(2): 333-336.

[10] Swartz PG, Roberts CC. Radiological reasoning: bone marrow changes on MRI. American Journal of Roentgenology. 2009; 193(3): S1-S4.

[11] Rahmouni A, Montazel JL, Divine $M$, et al. Bone marrow with diffuse tumor infiltration in patients with lymphoproliferative diseases: dynamic gadoliniumenhanced MR imaging. Radiology, 2003; 229(3): 710-717.

[12] Dutoit JC, M.A. Vanderkerken MA, Verstraete KL. Value of whole body MRI and dynamic contrast enhanced MRI in the diagnosis, follow-up and evaluation of disease activity and extent in multiple myeloma. European Journal of Radiology. 2013; 82(9): 1444-1452.

[13] Kwee TC, Kwee RM, Nievelstein RA. Imaging in staging of malignant lymphoma: a systematic review. Blood. 2008; 111(2): 504-516.

[14] Alyas F, Saifuddin A, Connell D. MR imaging evaluation of the bone marrow and marrow infiltrative disorders of the lumbar spine. Magnetic resonance imaging clinics of North America. 2007; 15(2): 199-219.

[15] Kwee TC, Kwee RM, Verdonck LF, et al. Magnetic resonance imaging for the detection of bone marrow involvement in malignant lymphoma. British Journal of Haematology. 2008, 141(1): 60-68.

[16] Vanel D, C. Dromain, Tardivon A. MRI of bone marrow disorders. European Radiology. 2000; 10(2): 224-229. 